\title{
Analytic approach to the equation of Esbjerg and Nørskov
}

\author{
A. N. Andriotis \\ Theoretical and Physical Chemistry Institute, National Hellenic Research Foundation, \\ 48 Vassileos Constantinou Avenue, Athens 116/35, Greece
}

(Received 5 September 1985)

\begin{abstract}
The equation of Esbjerb and Norskov, $E(z)=\alpha_{\text {eff }} \rho(z)$, is obtained analytically as the leading term of a functional $E[\rho(z)]$ which relates the interaction energy $E(z)$ between a helium atom at position $z$ from a metal surface with the electron density $\rho(z)$ of the unperturbed metal. It is shown that $\alpha_{\text {eff }}$ does not depend strongly on $\rho(z)$, but on the metal substrate. Furthermore, $\alpha_{\text {eff }}$ depends strongly on the approximation employed in evaluating the overlap integrals between the $1 s$ helium state and the one-electron states of the metal substrates. Results for the interaction of helium with $\mathrm{Li}(001), \mathrm{Na}(001)$, and $\mathrm{K}(001)$ semiinfinite metals are also presented.
\end{abstract}

In a recent work ${ }^{1}$ (to be denoted by $\mathrm{I}$ ), we have shown that the equation of Esbjerg and Norskov, ${ }^{2}$

$$
E=\alpha_{\mathrm{eff}} \rho_{0},
$$

which relates the energy change $E$ upon embedding a $\mathrm{He}$ atom into a metallic jellium of free-electron density $\rho_{0}$, appears as the leading term in a series representation of a functional $E=E\left(\rho_{0}\right)$. The proportionality constant $\alpha_{\text {eff }}$ was found to depend on the parameters of the $1 s$-electron orbital $\Psi_{a}(r)$ of the free $\mathrm{He}$ atom. In the particular case for which

$$
\Psi_{a}(r)=\left(\frac{2 \beta}{\pi}\right)^{3 / 4} e^{-\beta r^{2}}
$$

we have shown that within the coupled Hartree-Fock (CHF) approximation $^{3}$

$$
\alpha_{\mathrm{eff}}=\left[-\epsilon_{a}-\left(\frac{2 \beta}{\pi}\right)^{1 / 2}\right]\left(\frac{2 \pi}{\beta}\right)^{3 / 2},
$$

where $\epsilon_{a}$ is the eigenvalue of the $1 s$ state of the He atom. The contribution to $\alpha_{\text {eff }}$ which comes from the $\epsilon_{a}$ term has its origin in the assumption that the single-electron wave functions of the metal, $|\mathbf{k}\rangle$, in the presence of the He atom, are obtained from the unperturbed ones, $\left|\mathbf{k}_{0}\right\rangle$, by orthogonalizing the latter to the $\Psi_{a}(r)$ electron state of the unperturbed $\mathrm{He}$ atom, i.e.,

$$
|\mathbf{k}\rangle=\left|\mathbf{k}_{0}\right\rangle-S_{a \mathbf{k}}|a\rangle \text {, }
$$

where $S_{a \mathrm{k}}=\left\langle a \mid \mathbf{k}_{0}\right\rangle$. [We are going to use, equivalently, the symbols $|\mathbf{k}\rangle$ and $|a\rangle$ for $\Psi_{\mathbf{k}}(\mathbf{r})$ and $\Psi_{a}(\mathbf{r})$, respectively.] The other contribution to $\alpha_{\text {eff }}$, given by the second term of Eq. (3), has its origin in the exchange-energy contribution to the interaction between the $\mathrm{He}$ atom and the metal. ${ }^{4}$

In the present work, we shall present a similar attempt to express $\alpha_{\text {eff }}$ by an equation analogous to Eq. (3) in the case of a $\mathrm{He}$ atom interacting with a metal surface. This attempt aims at the investigation of the inhomogeneity effects of the electron density on $\alpha_{\text {eff. }}$. It will be shown that $\alpha_{\text {eff }}$ can be expressed (as in I) in terms of the characteristics of the $1 s$ electron state of the $\mathrm{He}$ atom. Also, in the present case, the explicit dependence of $\alpha_{\text {eff }}$ on the characteristics of the metal substrate will be derived. Furthermore, it will be shown that the substrate dependence of $\alpha_{\mathrm{efr}}$ is very sensitive to the approximation employed in evaluating the overlap in- tegrals $S_{a k}$.

We start from the expression for the repulsive interaction $E_{\text {rep }}^{(1)}\left(z_{i}\right)$ a $\mathrm{He}$ atom is subjected to at position $z_{i}$ from a metal surface and which is due to the orthogonalization process described by Eq. (4):

$$
E_{\text {rep }}^{(1)}\left(z_{i}\right)=\sum_{\mathbf{k}}\left[\epsilon_{\mathbf{k}}^{(0)}-V_{\mathrm{eff}}\left(z_{i}\right)-\epsilon_{a}\right]\left|S_{a \mathbf{k}}\left(z_{i}\right)\right|^{2},
$$

where $V_{\text {eff }}(z)$ and $\epsilon_{\mathbf{k}}^{(0)}$ are the single-electron potential and eigenvalues of the electrons of the unperturbed metal, respectively. It is assumed that the metal occupies the negative $z$ space and is approximated according to a method described elsewhere. ${ }^{5}$ The $\epsilon_{\mathrm{k}}^{(0)}$ term of $E_{\text {rep }}^{(1)}\left(z_{i}\right)$ appears in the corresponding expression arrived at by Harris and Liebsch $^{6}$ and is included here for later discussion. It is noted that

$$
\epsilon_{\mathbf{E}}^{(0)}=\left\langle V_{\text {eff }}(-\infty)\right\rangle+\frac{1}{2} k^{2},
$$

where $\left\langle V_{\text {eff }}(-\infty)\right\rangle$ denotes the average value of $V_{\text {eff }}(z)$ far from the surface inside the metal. In the case of the semiinfinite metal

$$
\Psi_{\mathbf{k}}(\mathbf{r})=\left(\frac{2}{\Omega}\right)^{1 / 2} e^{i \mathbf{k}_{\|} \cdot p} \xi_{q}(z),
$$

where $\Omega$ is the volume of the system and $k=\left(k_{\|}, q\right)$.

In the following we will express $\Sigma_{k}\left|S_{a k}\left(z_{i}\right)\right|^{2}$ in terms of the free-electron density $\rho(z)$ of the unperturbed metal. In establishing this relationship, two approximations will be discussed.

In the first approximation (which we will improve subsequently), it is assumed that $\xi_{q}(z)$ is constant over the range of the $\mathrm{He}$ atom at $z_{i}$; i.e., $\xi_{q}(z)=\xi_{q}\left(z_{i}\right)$. Within this approximation, it can be easily found that

$$
\begin{aligned}
& \sum_{\mathbf{k}}\left|S_{a \mathbf{k}}\left(z_{i}\right)\right|^{2} \\
& \quad=\frac{2 \beta}{\pi^{2}}\left(\frac{2 \pi}{\beta}\right)^{3 / 2} \int_{0}^{k_{F}} d q\left|\xi_{q}\left(z_{i}\right)\right|^{2}\left(1-e^{-\left(k_{F}^{2}-q^{2}\right) / 2 \beta}\right),
\end{aligned}
$$

where $\frac{1}{2} k_{F}^{2}=\epsilon_{F}$ is the Fermi energy of the metal.

If the exponent $\beta$ is not too small, then the exponential in the integrand of Eq. (8) can be approximated by the first 
two terms of the corresponding Taylor series, and then

$$
\begin{aligned}
\sum_{\mathbf{k}}\left|S_{a \mathbf{k}}\left(z_{i}\right)\right|^{2} & =\left(2 \frac{\pi}{\beta}\right)^{3 / 2} \frac{1}{\pi^{2}} \int_{0}^{k_{F}}\left|\xi_{q}\left(z_{i}\right)\right|^{2}\left(k_{F}^{2}-q^{2}\right) d q \\
& =\left(2 \frac{\pi}{\beta}\right)^{3 / 2} \rho\left(z_{i}\right) .
\end{aligned}
$$

This result indicates that the effect of the surface is to replace the $\epsilon_{a}$ term of Eq. (3) by the coordinate dependent term,

$$
\epsilon_{a}\left(z_{i}\right)=\epsilon_{a}+V_{\mathrm{eff}}\left(z_{i}\right)-\left\langle V_{\mathrm{eff}}(-\infty)\right\rangle .
$$

The derivation of Eqs. (9) and (10) was based on the assumption that the kinetic energy term $k^{2} / 2$, due to $\epsilon_{\mathbf{k}}^{(0)}$, can be neglected. This assumption, although it was proved satisfactory in the embedding problem, ${ }^{1}$ cannot be ignored here. Retaining the $k^{2} / 2$ term of $\epsilon_{\mathbf{k}}^{(0)}$, it can be shown that the $\left\langle V_{\text {eff }}(-\infty)\right\rangle$ term on the right-hand side (RHS) of Eq. (10) will be replaced by the work function $\Phi$ of the metal substrate.

Thus, within the approximation $\xi_{q}(z) \simeq \xi_{q}\left(z_{i}\right)$, the contribution $\delta \alpha_{\text {eff }}^{(1)}$, which is due to the orthogonalization procedure given by Eq. (4), is

$$
\delta \alpha_{\mathrm{eff}}^{(1)}\left(z_{i}\right)=\left(\frac{2 \pi}{\beta}\right)^{3 / 2}\left[-\epsilon_{a}-V_{\mathrm{eff}}\left(z_{i}\right)-\phi\right] .
$$

It is noted that in the absence of the work-function term from the RHS of Eq. (10), a case which refers to the CHF approximation $^{3}$ of the He-metal interaction, the repulsive interaction $E_{\text {rep }}^{(1)}$ has the same functional relationship $E_{\text {rep }}^{(1)}=E_{\text {rep }}^{(1)}\left[\rho\left(z_{i}\right)\right]$ for every metal, when $V_{\text {eff }}\left(z_{i}\right)$ is approximated within the local electron density approximation. On the other hand, if $\Phi$ is retained, it is clear that $E_{\text {rep }}^{(1)}\left(z_{i}\right)$ will be higher for a metal with a low work function than for a metal with a higher one. This is contrary to the expected result ${ }^{6}$ and, as will be shown later, is due to the approximation $\xi_{q}(z)=\xi_{q}\left(z_{i}\right)$.

The approximation made in going from Eq. (8) to Eq. (9) can be considered valid for all practical purposes as the Gaussian-type orbitals (GTO) which describe the $1 s$ state of the $\mathrm{He}$ atom are not too diffusive. In the case where a linear combination of GTO's is used to describe the $1 s$ state of helium (instead of using one GTO) Eq. (11) becomes

$\delta \alpha_{\mathrm{eff}}^{(1)}\left(z_{i}\right)=(2 \pi)^{3 / 2}\left[-\epsilon_{a}-V_{\mathrm{eff}}\left(z_{i}\right)-\phi\right] \sum_{i, j} \frac{N_{i}^{(0)} N_{j}^{(0)}}{\left(\beta_{i} \beta_{j}\right)^{3 / 4}}$,

where $N_{i}^{(0)}$ are the coefficients of the GTO's. These are determined by solving the Hartree-Fock equation for the free $\mathrm{He}$ atom.

In order to improve the approximation $\xi_{q}(z) \simeq \xi_{q}\left(z_{i}\right)$ which was employed in deriving Eq. (12), we take

$$
\xi_{q}(z)=\xi_{q}\left(z_{i}\right)+\left(z-z_{i}\right) \xi_{q}^{\prime}\left(z_{i}\right)+\frac{\left(z-z_{i}\right)^{2}}{2} \xi_{q}^{\prime \prime}\left(z_{i}\right)
$$

over the range of the $\mathrm{He}$ atom. Equation (13) is then used to determine $S_{a \mathbf{k}}\left(z_{i}\right)$ and subsequently the sum $\Sigma_{\mathbf{k}}\left|S_{a \mathbf{k}}\left(z_{i}\right)\right|^{2}$. The term of $\xi_{q}(z)$ which corresponds to its first derivative does not contribute to $S_{a k}\left(z_{i}\right)$. Including the term proportional to the second derivative of $\xi_{q}(z)$, Eq. (9) becomes

$$
\sum_{\mathbf{k}}\left|S_{a \mathbf{k}}\left(z_{i}\right)\right|^{2}=\left(2 \frac{\pi}{\beta}\right)^{3 / 2}\left(1-\frac{E_{q}\left(z_{i}\right)}{2 \beta}\right)^{2} \rho\left(z_{i}\right),
$$

where

$$
E_{q}\left(z_{i}\right)=\left\langle V_{\text {eff }}(-\infty)\right\rangle+\frac{1}{2} q^{2}-V_{\text {eff }}\left(z_{i}\right) .
$$

In the process of deriving Eq. (14), the $\frac{1}{2} q^{2}$ term of the RHS of Eq. (15) is replaced by its average value over the $\left[0, k_{F}\right]$ interval.

As $E_{q}\left(z_{i}\right)$ is negative, Eq. (14) indicates that by including second-order corrections to the eigenfunctions $\xi_{q}(z)$, the repulsive interaction between the $\mathrm{He}$ atom and the metal appears stronger than without these corrections. Furthermore, Eqs. (11), (14), and (15) predict that the repulsive interaction $E\left(z_{i}\right)$ is stronger the higher the work function of the metal is (in the small-overlap region). This conclusion is reversed as $V_{\text {eff }}\left(z_{i}\right)$ tends to $\Phi$. It is understood that this comparison refers to the case where a $\mathrm{He}$ atom probes the same electron density in different metals and under the approximation $\frac{1}{2}\left\langle q^{2}\right\rangle=\epsilon_{F}$. More accurately, $\left\langle q^{2}\right\rangle=\epsilon_{F} / 5$ over the Fermi sphere. This latter approximation is used in our numerical results.

In the case where a linear combination of GTO's is used to describe $\Psi_{a}(r)$, Eq. (14) takes the following form:

$$
\sum_{\mathbf{k}}\left|S_{a \mathbf{k}}\left(z_{i}\right)\right|^{2}=(2 \pi)^{3 / 2} \sum_{i, j} \frac{N_{i}^{(0)} N_{j}^{(0)}}{\left(\beta_{i} \beta_{j}\right)^{3 / 4}} \rho\left(z_{i}\right)\left(1+\frac{E_{q}^{2}\left(z_{i}\right)}{4 \beta_{i} \beta_{j}}-\frac{E_{q}\left(z_{i}\right)\left(\beta_{i}+\beta_{j}\right)}{2 \beta_{i} \beta_{j}}\right) .
$$

Finally, following the same procedure as in $I$ and using the expression found for the exchange interaction between a He atom and a metal surface, ${ }^{3}$ we find the exchange-energy contribution to $\alpha_{\text {eff. }}$ This is given approximately by the expression found in the case of $\mathrm{He}$ embedding (paper I), multiplied by the factor which appears within the large parentheses on the RHS of Eq. (16).

Thus we are able to write down an analytic expression for the coordinate-dependent constant $\alpha_{\text {eff }}\left(z_{i}\right)$ which generalizes the constant of the equation of Esbjerb and Norskov:

$$
\alpha_{\mathrm{eff}}\left(z_{i}\right)=\sum_{i, j} N_{i}^{(0)} N_{j}^{(0)}\left(-\frac{2^{5 / 2} \pi}{\left(\beta_{i} \beta_{j}\right)^{1 / 4}\left(\beta_{i}+\beta_{j}\right)^{1 / 2}}-\frac{(2 \pi)^{3 / 2} \epsilon_{a}\left(z_{i}\right)}{\left(\beta_{i} \beta_{j}\right)^{3 / 4}}\right)\left(1+\frac{E_{q}^{2}\left(z_{i}\right)}{4 \beta_{i} \beta_{j}}-\frac{\left(\beta_{i}+\beta_{j}\right) E_{q}\left(z_{i}\right)}{2 \beta_{i} \beta_{j}}\right) .
$$

In Fig. 1 we indicate the variation of $\alpha_{\text {eff }}(z)$ given by Eq. (17) as a function of $\rho(z)$ for the cases of He interacting with the metals $\mathrm{Li}(001), \mathrm{Na}(001)$, and $\mathrm{K}(001)$. The computed value of $\epsilon_{a}$ is -0.918 a.u. We used the self-consistently obtained values for $V_{\text {eff }}(z)$ and $\rho(z)$ of Ref. 7. The work function $\Phi$ is $3.56,3.08$, and $2.59 \mathrm{eV} \mathrm{for} \mathrm{Li}(001), \mathrm{Na}(001)$, and $\mathrm{K}(001)$ semi-infinite metals, respectively. In the present calculations we made the approximation $q^{2} \simeq\left\langle q^{2}\right\rangle=\epsilon_{F} / 5$ for the $q^{2}$ term on the RHS of Eq. (15). If $E_{q}\left(z_{i}\right)$ were put identically equal to zero in Eq. (7) [i.e., employing the approximation 


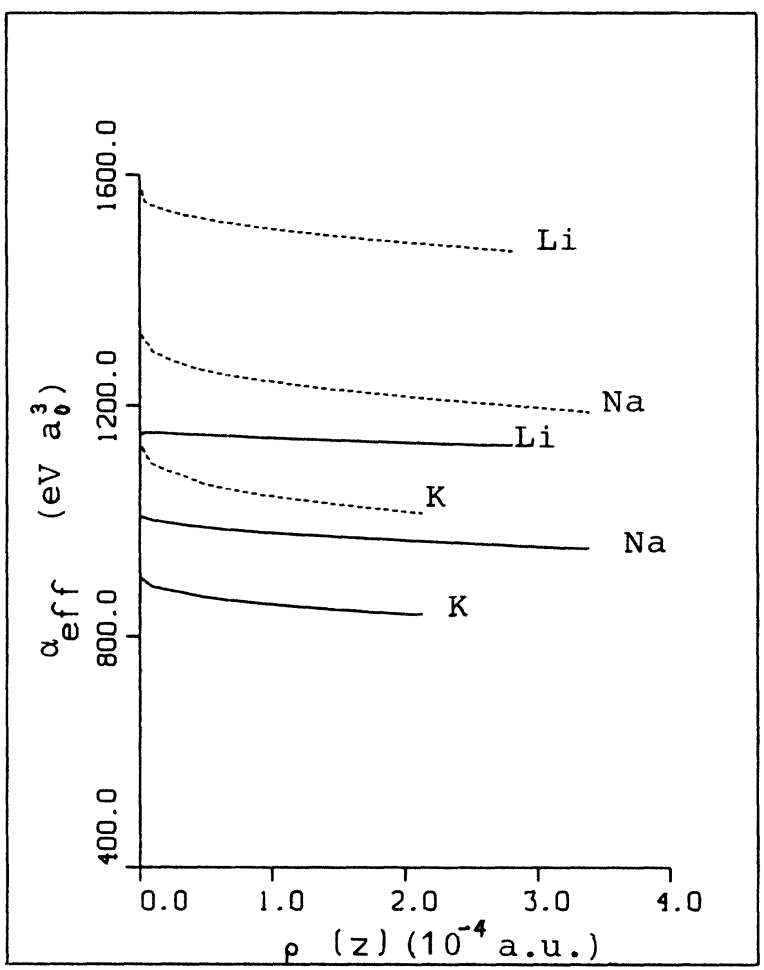

FIG. 1. The functional $\alpha_{\text {eff }}(z)=\alpha_{\text {eff }}[\rho(z)]$ as given by Eq. (17) (solid curves) for the cases of $\mathrm{He}$ interacting with the semi-infinite metals $\mathrm{Li}(001), \mathrm{Na}(001)$, and $\mathrm{K}(001)$. The 1 s-electron state of the free $\mathrm{He}$ atom is calculated within the Hartree-Fock approximation by expanding it in seven GTO's. For the metal substrates we use the self-consistently obtained values of $\rho(z)$ and $V_{\text {eff }}(z)$ of Ref. 7 . The dashed curves correspond to the case of the CHF approximation (Ref. 3), i.e., for the case $\epsilon_{a}(z)=\epsilon_{a}+V_{\text {eff }}(z)$.

$\left.\xi_{q}(z) \simeq \xi_{q}\left(z_{i}\right)\right], E(z)$ will appear (not shown here) stronger the lower the work function of the metal substrate is. However, the approximation given by Eq. (13) reverses this trend. This is shown in Fig. 1, where the solid curves represent Eq. (17) with $\epsilon_{a}\left(z_{i}\right)$ given by the second factor of the RHS of Eq. (11), and the dashed curves indicate the CHF limit [i.e., the case where $\epsilon_{\mathbf{k}}^{(0)}$ is set equal to zero in the RHS of Eq. (5)].

In Fig. 2 we indicate the variation of the repulsive interaction $E(z)=\alpha_{\text {eff }}(z) \rho(z)$ as a function of $\rho(z)$. It is observed

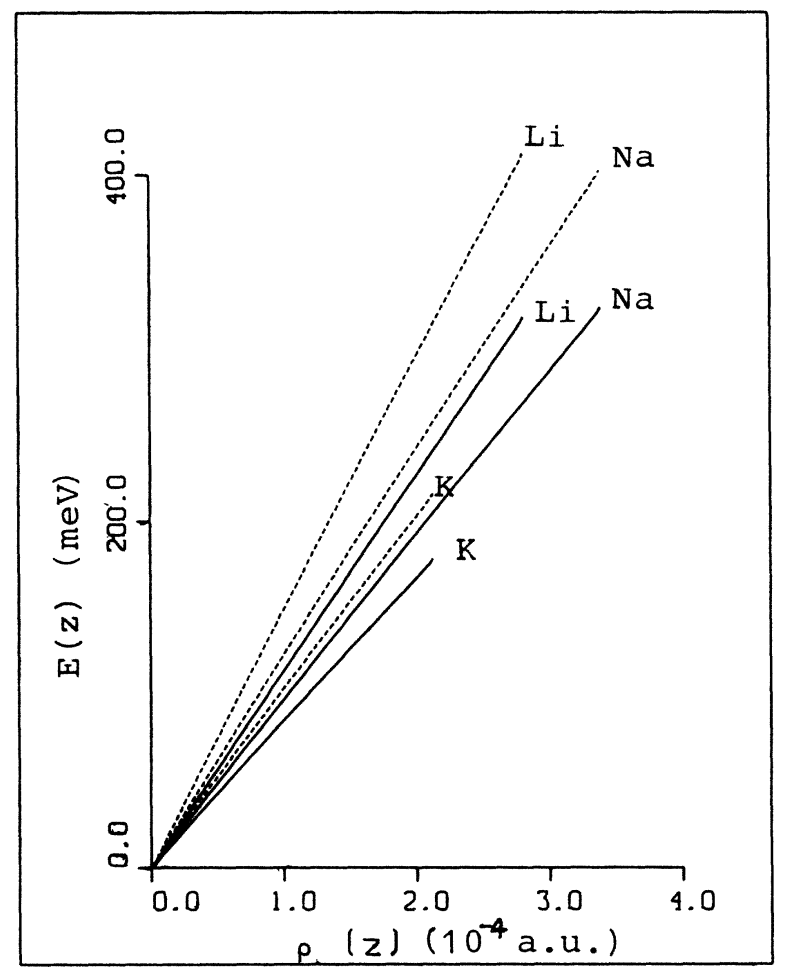

FIG. 2. The variation of the repulsive interaction $E(z)$ $=\alpha_{\mathrm{eff}}(z) \rho(z)$ as a function of the free-electron density $\rho(z)$. Solid (dashed) lines correspond to solid (dashed) curves of Fig. 1.

that in either case, that of neglecting the work function from $\epsilon_{a}\left(z_{i}\right)$ (dashed curves), or that of retaining the workfunction term (solid curves), the interaction $E(z)$ exhibits a strong substrate dependence. The density dependence of $E(z)$ is approximated by the linear relationship given by the generalized equation of Esbjerg and Norskov, i.e., Eq. (1). Thus, we can say that our results support the applicability of the generalized equation of Esbjerg and Norskov in the case of the interaction of helium with metal surfaces. The nonlinear [in $\rho(z)]$ terms of $E[\rho(z)]$, although they contribute significantly to the interaction $E(z)$, do not affect the linear relationship given by $\mathrm{Eq}$. (1). This behavior is due to a mutual cancellation between the nonlinear terms associated with $\delta \alpha_{\text {eff }}^{(1)}\left(z_{i}\right)$ given by Eq. (12) and with $E_{q}\left(z_{i}\right)$ given by Eq. (15)
${ }^{1}$ A. N. Andriotis (unpublished).

${ }^{2}$ N. Esbjerg and J. K. Norskov, Phys. Rev. Lett. 45, 807 (1980).

${ }^{3}$ A. N. Andriotis and C. A. Nicolaides, Solid State Commun. 51, 251 (1984).
${ }^{4}$ A. N. Andriotis, Phys. Rev. B 31, 4003 (1985).

${ }^{5}$ A. N. Andriotis, Surf. Sci. 116, 501 (1982).

6J. Harris and A. Liebsch, J. Phys. C 15, 2275 (1982).

${ }^{7}$ A. N. Andriotis, Phys. Rev. B 32, 5063 (1985). 